Corrigendum

\title{
Corrigendum to "Adaptive Nonlinear Tire-Road Friction Force Estimation for Vehicular Systems Based on a Novel Differentiable Friction Model"
}

\author{
Zhi-Jun Fu, Wei-Dong Xie, and Xiao-Bin Ning \\ Vehicle Engineering Research Institute, College of Mechanical Engineering, Zhejiang University of Technology, China \\ Correspondence should be addressed to Zhi-Jun Fu; fuzhijun@zjut.edu.cn \\ Received 1 September 2015; Accepted 18 October 2015 \\ Copyright (C) 2015 Zhi-Jun Fu et al. This is an open access article distributed under the Creative Commons Attribution License, \\ which permits unrestricted use, distribution, and reproduction in any medium, provided the original work is properly cited.
}

In the paper titled "Adaptive Nonlinear Tire-Road Friction Force Estimation for Vehicular Systems Based on a Novel Differentiable Friction Model" [1], there are two spelling mistakes in the paper as follows:

(1) $F_{-r f}$ in equation (2) and equation (3) should be $F_{-d}$.

(2) $+F_{d}$ in equation (4) should be $-F_{-\mathrm{rf}}$.

\section{References}

[1] Z.-J. Fu, W.-D. Xie, and X.-B. Ning, "Adaptive nonlinear tireroad friction force estimation for vehicular systems based on a novel differentiable friction mode," Mathematical Problems in Engineering, vol. 2015, Article ID 201062, 7 pages, 2015. 


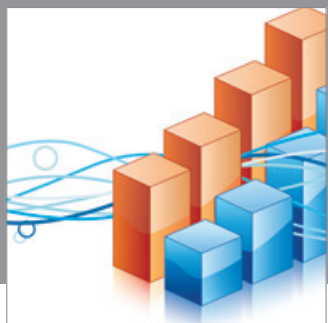

Advances in

Operations Research

mansans

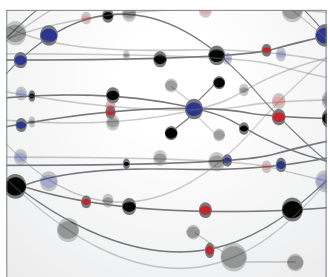

The Scientific World Journal
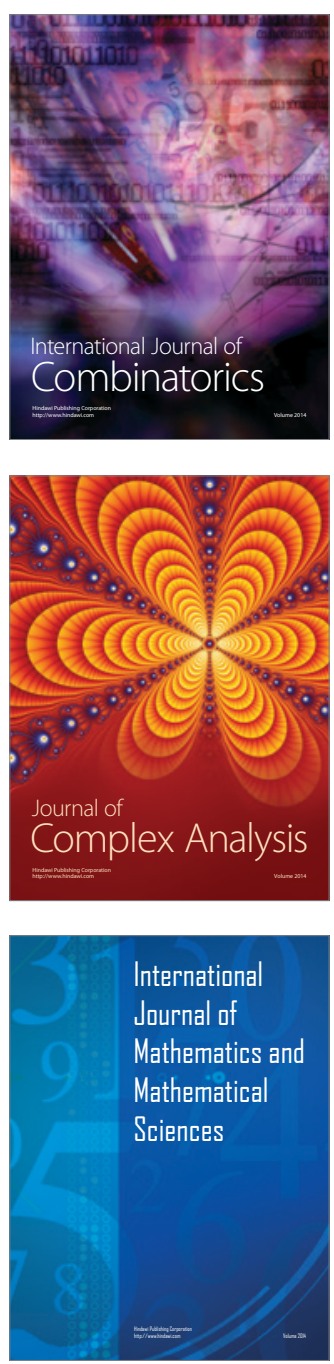
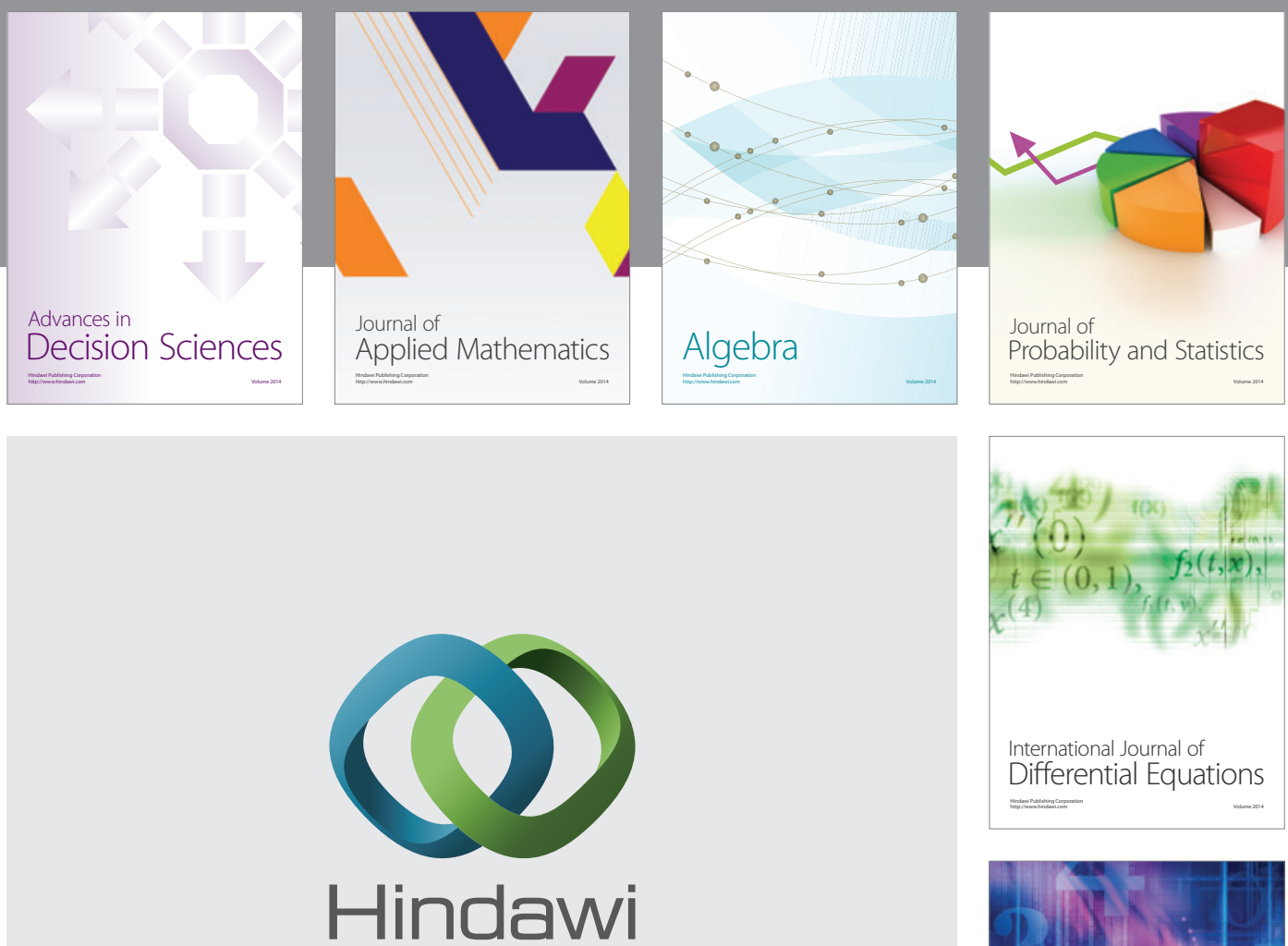

Submit your manuscripts at http://www.hindawi.com
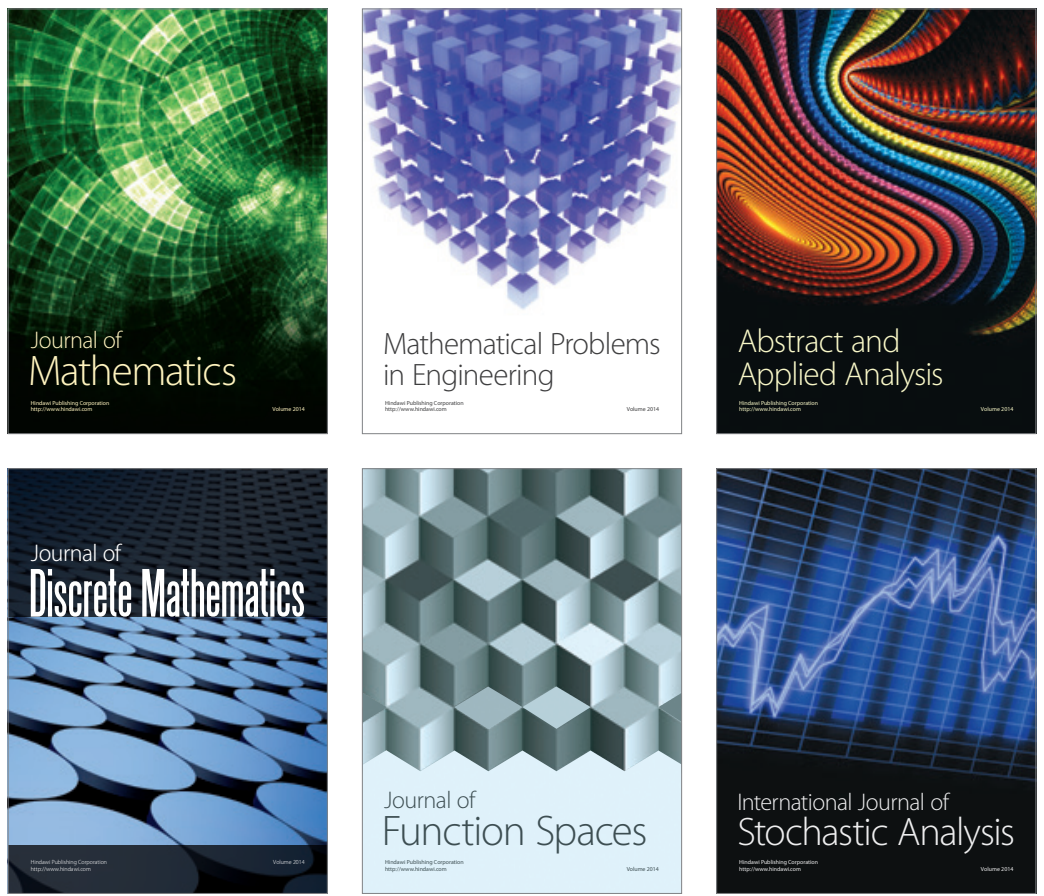

Journal of

Function Spaces

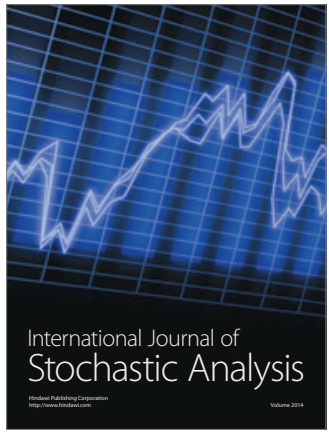

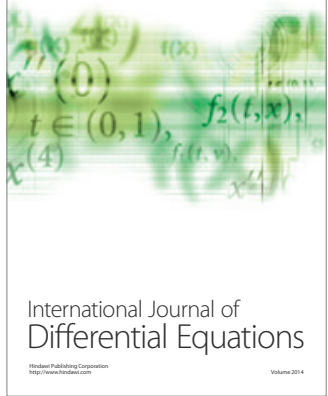
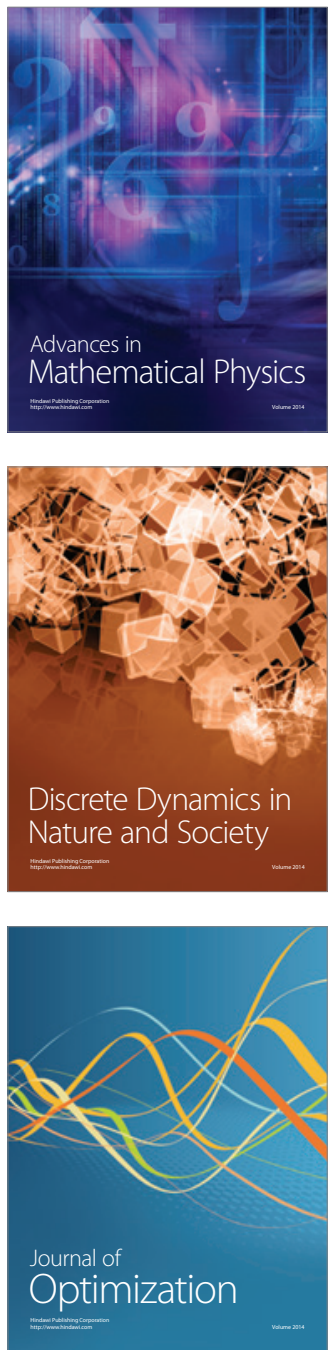